从阿中文化特点与传统看中文翻译与研究策略

\title{
On a Strategy of chinese Linguistic \& Translation studies from the perspective of Sino-Arabic cultural
} characteristics $^{(*)}$

\author{
Mohsen Fergani \\ Ain-Shams University, Al-Alsun, \\ Chinese language department, Abbasya, Cairo
}

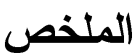

حول استراتيجية بحث في اللغة الصينية والترجمة من منظور الخصائص الثقافية العربية و الصينية

موضوع البحث يدور حول رؤية ينصور ها الباحث لاستر اتيجية تقوم عليها جهود البحث

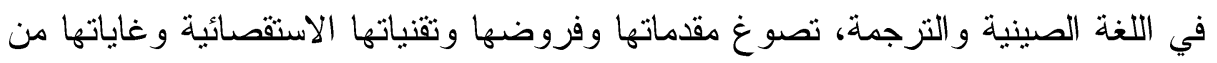

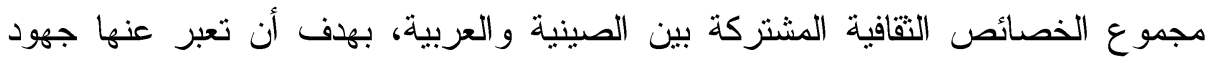

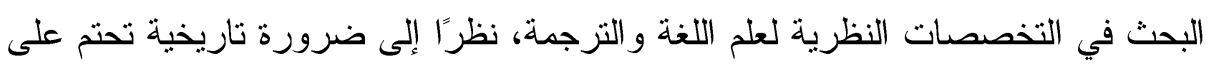

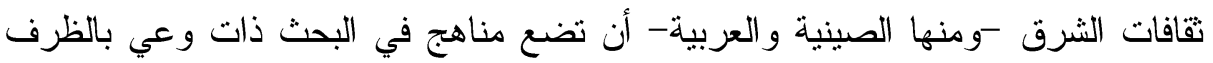

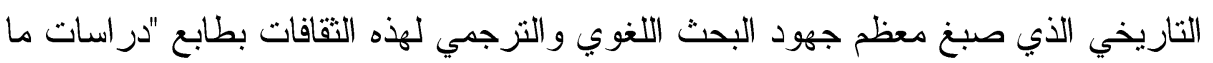

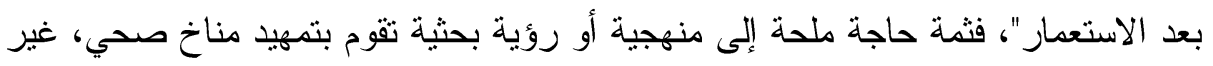
متأثز بخطاب الهيمنة الاستعمارية الموروث عن علاقات مأزومة بالغرب، تمهيدًا لأجواء الهاء

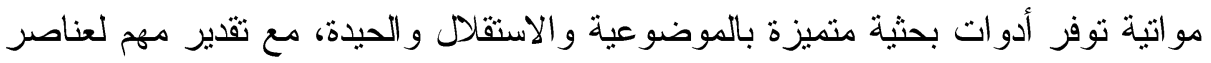
التقاء ثقافي شرقي، تنكلت عبر سنوات من التبادل التاريخي، وشكلت معها رصيدًا يمكن الاستتاد إليه في تقدير منظور جديد للترجمة والدرس اللغوي، وهو الرصيد الذي يمكن أن يصحح أو يوازن من المعايير التي جرت صياغتها في ظل خطاب دراسات مابعد الاستعمار، بما انطوي عليه من علاقات غير متكافئة في ميزان القوى الثقافية ومجموع خصائصها الحضارية.
\end{abstract}

${ }^{(*)}$ Bulletin of the Faculty of Arts Volume 79 Issue 2 January 2019 


\section{Abstract \\ On a Strategy of chinese Linguistic \& Translation studies from the perspective of Sino-Arabic cultural characteristics}

This paper presents a new perspective for building a theoretical basis for a strategy of linguistic and translation theory, to the arabic scholars and translators. A theory which derives its main visions and contents from the characteristcs of Sino-Arabic cultural traditions, with a view on rerranging the research field, in this category of theoretical studies, to include a new trend in Linguistic and traslation activities, instigating a developed narrative that would substitute the old set of ideas included in "Postcolonial transltion theories". The tranditional wealth of the Great Orient, which includes Arabia and china, will serve as a common reservoir that has shaped a long lasting fund for traditional evaluation system, and an everlasting criteria that works as a great common denominator of public sense in the Sino-Arabic mutual context of hisorical relations.

我之所以选择把中国文学作品译成阿语的一个最重要的原因在于，把此方面 的翻译活从语言层面上改为文化, 并使译语读者和译者能在阅读和写作上意识 到中国民族伟大的独特性, 同时也要避免转译以及其随伴过来的全球化的不对 称的权力矢系, 从这点上来讲, 翻译活动堪称一种政治行为。而中国文学作品 译成阿语, 在某种意义上, 翻译的作用要释放出来, 这种释放可以当做一项“翻 译策略”, 而在走出语言学的翻译研究范式之后, 笔者已经认识到语篇中的翻译 选择受到各种外部因素的影响和制约, “政治策略”是来自政治文化的, 那么政 治文化也是影响和制约翻译过程的一个很中要的因素。出于好几种原因, 笔者 会采取各种可能的策略来处理译中涉及的与政治文化相关的因素。

一、古老东方国家与全球化的影响

许多文学翻译工作者, 无论来自阿拉伯地区还是中国, 其翻译技能与理论观 点都受到全球化及其带来的思想成果的影响, 也有不少对翻译思考和研究方法 来自全球学术话语。由于根深蒂固的欧洲文化中心观念, 这些思想具有公认的 地位。然而在古老东方国家的悠久文化交流史上进行的翻译活动, 也有其悠久 
的积淀。它也应该在多种因素交错作用的翻译过程中, 构成批评、审美和思想 要素, 决定文学作品的接收和接受条件。这些要素超越了现代社会所定义的译 者的个人作用。长久以来通过自发性的口头创作, 文化交流活动创造了文学作 品的本土化形式。得益于这种自发性的创造, 交流被赋予了价值, 它能为人们 在文学接受过程中制定审美标准。比较文学研究者能够精确地追溯其发展过程 , 并得出有价值的重要结论, 用以解释其对伟大东方文明的社会、艺术、审美 等方面的影响。

在杰出的中国文学家季羡林先生

1 的比较文学研究成果中可以找到例证, 其研究成果揭示了翻译对东方不同文 明之间文化交流的重要影响。其中最重要的影响体现为：在印度、中国、波斯 及阿拉伯文明中, 人们通过翻译为文学作品的本土接受创造了条件, 为审美艺 术活动提供了创造动力。例如在季先生的研究中我们发现, 古印度故事集《五 卷书》在艺术审美方面对中国古代民间故事的创作产生了影响。《五卷书》确 实没有以整本书的形式传入中国, 但是其中的一些寓言、童话和小故事, 确亦 传入中国, 在中国民间故事以及文人的创作中, 在极其悠久的历史上, 蛛丝马 迹, 确能寻出。而文化关系研究者则可能发现翻译更深层次的影响, 即在民间 故事创作风格上形成无形的东方文化中心主义审美特征。我认为, 长久以来这 种文化中心主义就是翻译潜移默化造成的。或许这种文化中心主义构成了东方 文明的总体审美品位, 又或许它仍然是中国和阿拉伯地区文学作品接受的条件 。在此基础上形成了东方民族和人民根本的审美标准。我们知道, 《五卷书》 的阿拉伯语译本名为《卡里来和笛木乃》, 该译本体现了阿拉伯审美趣味。或 许通过分析阿拉伯人的《卡里来和笛木乃》与中国人的《黔之驴》的翻译过程 , 我们就能清楚找到中阿共同的审美要素。当然《卡里来和笛木乃》对许多其 他文化中的民间故事创作产生了重要影响, 但是译者伊本.穆加法为阿拉伯语译 本添加了一些审美要素, 这为阿拉伯人理解、解释、创作故事提供了新的创造 性价值。正如中文翻译使唐朝的民间故事创作得到发展, 印度文学在元朝对中 国的戏剧创作起到间接影响, 波斯故事集《赫扎尔—

艾福萨那》也曾经对《一千零一夜》的叙事风格产生影响。

“《五卷书》的名声在公元 6 世纪时波斯。波斯国王命令医生“白尔才外”把 此书的一个西北传本译为中世波斯文言巴利维语。一个由此本译出来的古代叙 利亚语本和一个阿拉伯语本却保存下来。750年出现的一个伊本.阿尔穆加法

'中国学者季羡林性于1911年, 1934年毕业于清华大学西洋文学系, 是五十年代中期至八十年代 中期最重要的此较文学研究者之一, 于2009年逝世。 


\section{4}

Bulletin of the Faculty of Arts Volume 79 Issue 2 January 2019

Ibn

Al-Muqaffa

译出来的阿拉伯文本, 增加了许多原书没有的新材料, 书名也叫《卡里来和笛 木乃》。这个阿文译本成了以后不断出现的欧亚众多语言译本的来源... ...

19 世纪德国Theodor

Benfey

把此书译为德文, 在他的著名的长篇导言中, 他以惊人的博学多识, 通过惊人 多的语言, 追踪了《五卷书》在世界上的流传演变的过程 ... $\quad \ldots$

他还主张此书中的绝大部分故事来源于佛教。”2

学者们对于《一千零一夜》故事的文化源头各执已见，但这种分歧恰好证明 了民间故事叙事方式在翻译时受到了多种东方文化的影响。这表明在翻译中, 深人人心的审美标准或长期存在的民族文化特征包容了多种风格, 以对故事的 叙述进行解释和再创造, 而并非只认同一种权威性的形式, 用一套僵化的标准 来衡量创作, 限制不同的审美创作风格。幸运的是, 东方文明并不认为其文学 作品因具有特色就应当成为被世界效仿的典范, 并冠以“世界文学”名号, 正如1 8 世纪德国人歌德所说的德国文学那样。

二、东方文明中的翻译做为保卫文化独特性的工具

东方文明中的文学翻译方法不同于西方欧美文化中人类思想所创造的现代翻 译方法, 东方翻译的传统有着深刻的审美理念, 它包容了多种形式的文学遗产 , 并创造性地对其进行增补, 从而以全新的视角创造一种能够引发争议、进行 创新、解释人类矢系和现象的灵活思维方式。笔者认为, 中阿文译者应当从东 方文明之间的历史交流情况出发, 构建两种文化之间的文学翻译观。或许可以 观察并发掘每个地区的文化特色, 这种特色让各个地区都有权从事创造、创新 ，而不受某一种僵化的审美艺术创作模式的束缚。

笔者并非质疑西方文明在科研、理论、技术运用领域为文学翻译做出的伟大 贡献, 它提供了强大的交流基础, 并为发展和现代化创造了重要条件。比较欧 洲和中国翻译的历史, 以及翻译的理论, 可以看出, 东西双方探讨的问题基本 相同。双方都讨论翻译的可能与不可能的问题, 也讨论直译与意译的问题。但 是双方也有不同之处。西方谈翻译理论, 偏重于可能与不可能的问题, 以及可 能的程度。他们得出的结论是: 文学翻译难, 科技翻译易。直译与意译问题, 也偶尔涉及 ; 但不是重点。在翻译的基础或背景方面, 欧洲与中国甚至于阿拉 伯不同。在欧洲, 除了最早的希伯来语以外, 基本上是同一语系的语言之间的 互相翻译。因此才产生了某一些理论家主张的翻译三分法：

${ }^{2}$ 季美林 《比较文学与民问文学》, 北京: 北京大学出版社, 1991 年, 页345。 


\section{一、翻译；}

二、变换；

\section{、逐词对照本。}

这种三分法对中国或其它东方古国是完全不适用的。中国绝不可能有变换。 因为在中国几千年的翻译史上都是不同语系语言之间的翻译, 在同一语系语言 间才能变换。中国偏重于直译与意译之争, 所谓文与质者就是。这是由于从佛 经的翻译到现代科学文学着作的翻译, 都有其特殊的文化和历史背景。

然而, 西方对宗教神学和哲学作品的部分翻译, 以及西方的现代学术传统, 也限制了翻译的创造力。或许将翻译方法区分为“忠实”和“背叛”的严格的二元 论, 印证了中心模式的主导地位, 这种模式在不同文化中对文学翻译的方式、 概念和传统施加影响。最好的例证是, 美国语言家乔姆斯基在翻译的研究与应 用上传承了卡特福德, 具有明显的传教目的。即使是尤金.奈达, 其构建的科学 翻译理论思想也将翻译的功能与 《圣经》的宗教阐释混为一谈。直到 20 世纪 80 年代，西方的翻译研究基本上都只限于语言层面的分析！

总体而言, 在欧洲中心观念的背景下, 翻译理论和观念在西方建立了起来, 其中大部分都源于希腊罗马传统、基督教价值观念及国家主义的愿景。笔者认 为有必要重新评估与审视这些理论, 但目前更应当认真思考如何确立中阿翻译 的基本观念和思想。重要的并非要上升到批评理论层面, 而是应通过探究两种 文化之间的交流史, 建立中阿双方矢于互译的共同观念, 从而使学者拥有新的 理论框架, 它应与西方中心所创造的理论不同, 后者只是西方历史背景和社会 传统的产物。据我所知，中国研究界轓经探讨有矢翻译文化的学术问题，“中国 当代翻译界, 明确地提出运用文化学的观点来审视翻译问题的, 较早的一篇文 章是刘山先生发表在1982年第5期《翻译通讯》（即《中国翻译》前身）的《 翻译与文化》。这篇文章指出: 翻译是不同民族沟通思想、交流文化的行为。 它诚然要通过不同民族语言文字的转换来实现, 但这种转换并不是、或不只是 寻求语言的等值, 而是要通过一种民族语言去忠实地反映（或恰当地再现）另 一民族的思想和文化。”

接着, 王佐良先生在1985年第1期《翻译通讯》上发表的《翻译与文化繁荣》 一文中, 明确倡导翻译研究不能忽略社会文化因素。他认为翻译里最大的困难 就是两种文化的不同。在一种文化里头有一些不言而喻的东西, 在另一种文化 里头却要费很大力气加以解释。他在1989年发表的《翻译中的文化比较》一文

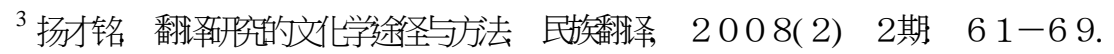


中又进一步指出：翻译者必须是一个真正意义的文化人。他认为翻译中真正的 对等应该是文化含义的对等, 应该在文化作用、范围、情感色彩、影响等方面 都相当。

笔者认为, 中国文学的阿拉伯语翻译迫切需要形成一种向“王佐良”先生所提 出的那样建立在社会文化因素和社会独特性的理论框架, 表达民族特点, 即 :

1、对阿拉伯民族个性进行定义, 体现阿拉伯地区在与东方发生矢系时的历 史、心理与社会特征；

2、阿拉伯的审美艺术表达方式形成了矢于阿拉伯语翻译的标准或特征, 有 必要对这些表达方式进行阐释。中国在现代已多次尝试为其翻译活动构建理论 基础。上世纪三十年代, 林语堂等人尝试建立具有中国特色的翻译学。季羡林 先生曾尝试在 “东方文学”研究领域提出一种中国理论, 矢注制定理论观点、研 究方法、实践途径, 以吸取文学创作中的世界优秀经验。这位在比较文学研究 领域的前辈学者认为, 西方的殖民主义已经将“东方”一词由地理历史概念转变 成了政治概念。笔者认同此观点。因此, 任何矢于阿拉伯语翻译的理论都应当 从战略角度出发, 并应充分考虑欧洲中心主义给东方文化的定位。

笔者作为中国文学的译者和中国文化的研究者, 并且身为阿拉伯人, 认为应 以当前中阿矢系的总体氛围为契机, 构建翻译理论, 这意味着赋予阿拉伯语翻 译工作价值, 尊重译者的创造性, 展示历史进程中形成的阿拉伯文化特点, 同 时尊重阿拉伯语翻译过程的独立性, 包括翻译方法、翻译对象选择、文化奖励

、评价标准、审美艺术的表达方式, 以及在翻译中文作品时有助于再创造的道 德、社会与心理价值观念。看目前情况, 文学翻译很可能有助于阿中两民族在 转换过程中比此的文化独特性。

笔者认为, 可以从中国“一带一路”倡议 4 中受益, 从东方文明之间的文化相近出发, 来构建中阿文学翻译工作的基本 思想和可行观点。这能给阿拉伯语翻译工作带来优势, 即从阿拉伯历史上的翻 译经验中波取灵感, 而不受在殖民文化背景下制定的翻译理论限制, 不被僵化 观念下产生的传统文化所羁绊, 这些观念只不过是基于欧洲的历史和创作条件 来看待文学作品。

4“一带路”倡议由习近平主席于2013年9月10日提出。指的是: 1、给绸之路弪剂带, 2、21世纪 海上丝绸之路。旨在与些俴家建立多方位合作机制, 该昌议借用了“丝绸之路”这一历史名词, 高举

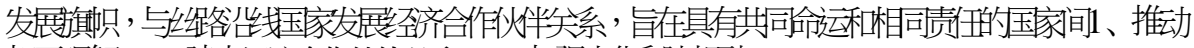
相互理解，2、建立经济合作火火半关系，3、加强文化和旺相融。 
文学翻译中, 当译者作为原作的理解者彰显主体性地位的时候, 其译作已然 不再是原作的忠实再现, 而是成为文学作品的一种存在形式, 是以原作意义为 基础的背离。与此同时, 具有独立身份的译者还会向目的语读者发出邀约, 邀 请他们带着自身的视域走进文本的视域进行理解, 去填补“不定点”和“空白”, 并召唤一切由视域融合而产生的新意义, 努力发掘译作中的潜在价值, 使其成 为现实价值。阿中两种文化, 长久以来所沉积了过去一代代人所积累的经验, 能 有助于弥步此“空白”同时能脱离译者的“主体性”, 另外, 这个主体性, 原来并 不是人的自主性和能动性, 而是人的社会性和历史性, 是人的基本历史活动一

劳动, 而劳动是不为人的主观愿望左右的、自为存在的活动。文学翻译话语建 构不是一种孤立的语言转换活动, 作者、译者、读者和目的语接受环境中的任 何一个主体均不能统摄整个话语事件, 都不是绝对的主体, 而是以对方存在为 前提的一种共在的自我。从这点上来讲, 东方文明中之间的对化可以通过文学 翻译构建两种文化之间的文学翻译观

, 还或许可以观察并发掘每个地区的文化特色, 这种特色让各个地区都有权从 事创造、创新，而不受某一种僵化的审美艺术创作模式的束缚。

三、阿拉伯语言学者要分清“语言学”与“翻译学”之间的界限

翻译学应该被视为一门独立的学科, “就像语言学和文学研究一样独立于其 他门类的学科。”

因为翻译具有特别的特性, 如果再考虑翻译学的语言方面、美学方面和文化内 涵等因素, 我们就不能将翻译学视为一个严格的人文学科分支, 因为翻译学还 融入了文化的因素, 所以承载着文化阐释的功能。作为一门的学科, 翻译学应 该具有自己独特的研究对象、研究领域和方法论, 同时, 还应该包含所有翻译 实践中的问题和翻译研究中的理论。另外, 在文化语境下进行的翻译研究可以 得益于各种现代理论的支持, 它是以所有被压抑的边缘化语、亚文化和文学中 的亚文类的解放为标志。基于此，如果把阿拉伯翻译活动融入文化翻译之中, 那么它很容易受到“后殖民主义翻译研究”重陶，因位阿拉伯文化曾经遭受到同 西方征服者的文化权力上的不平等的对话。而如今的阿拉伯翻译活动是长期以 来沦为此文化状况，很可能离不开思想材料的长期积累，）

正是凭着这一紧迫而崇高的学术责任感,该文章坚持把中国最近提出的“一带一 路”倡议当做一项有助于脱离强势文化和弱势文化所构建的权力话语中的不平等

${ }^{5}$ 王宁〈文化翻妇与经典阐释》, 中华遍, 2006年, 
的文化对话、新的文学翻译理论的想征。

笔者认为, “一带一路”倡议在文化层面能够提供一个有利环境, 以展示阿拉 伯语翻译的创造性特色与传统, 这些传统曾与印度、波斯作品謧美, 并在文本 中加入了原汁原味的阿拉伯特色, 而且已经证明它能够历久弥新, 并在阐述人 类长期的经验活动方面具有恒久性价值。阿拉伯语翻译作品的特色能够在世界 不同的文化中传播便是证明。笔者认为, 海上与陆上丝绸之路沿线汇集了多种 文明, 这能够为不同类型的文学翻译提供强大动力, 包容东方人民所有的人文 特色并体现其价值, 强调不同文学的叙事艺术的创造性价值。

最后, 我觉得我们阿拉伯学者能从“一带一路”倡议以及从中国经验中获得启 迪, 提出平行的阿拉伯文化策略, 尤其是在文学翻译领域提出可以付诸实践的 观念。笔者认为, 这一问题已不是一种 (可有可无的) 奢侈思想, 也不仅仅是 一种设想, 而是为应对西方殖民主义影响的必要举措。在西方殖民主义的影响 下, 全世界的文学创作遵照着一种中心模式, 因此文学交流的评价标准充满了 疑虑、紧张和痛苦, 而东方文化却被边缘化。或许, 一直以来任何一种证明自 身优越性的文化, 都会受到质疑, 那么如果抱着西方文明优越的成见来接受中 国文学作品, 那将是不幸的。总之, 对于从事中国文学翻译的阿拉伯译者和学 者来讲, 最重要的一个责任, 就是要拓展自己的研究视野, 同时还要放弃以往 的翻译理论多为建立在对语言进行分析的基础上,

而后殖民主义翻译理论则摆脱了单一语言研究的束缚,

运用政治学、社会学、文化学、人类学、文学、哲学、历史和心理分析等领域 的研究成果, 通过分析来自不同文化的文本和行为,

构筑了研究中国语言与文学翻译的策略。 


\section{参考文献}

[1]张柏然, 刘华文, 张思洁.中国译学:传承与创新.上海: 上海外语教育出版社, 2 008.

[2]王宁.文化翻译与经典阐释.北京:中华书局, 2006.

[3]薛庆国选集,北京外国语大学70周年校庆学术成果系列.外语学院与研究出版 社,2011.

[4]孙建成.水浒传英译的语言与文化.上海: 复旦大学出版社,2008.

[5]程裕祯.中国文化要略.北京:外语教学与研究出版社,2003.

[6]汪榕培,李正栓.典籍英译研究.河北大学出版社,2005.

[7]丛滋杭.中国古典诗歌英译理论研究.北京: 国防工业出版社,2007.1.

[8]季羡林.比较文学与民间文学.北京: 北京大学出伴社,1991.

[9] 扬才铭. 翻译研究的文化学途径与方法. 民族翻译, 2008 (2) 2 期.

[10] 吴国玢, 戴炜华."论语言学的跨学科研究", 上海理工大学,外语学院, 2012,一期.

[11]习近平谈治国理政.北京:外文出版社,2014.

[12]2014年《咬文嚼字》合定本.上海:上海锦绣文章出版社,2015. 